\title{
DECOMPOSIÇÃO E LIBERAÇÃO DE NUTRIENTES DA SERAPILHEIRA FOLIAR EM UM TRECHO DE FLORESTA OMBRÓFILA DENSA ALUVIAL EM REGENERAÇÃO, GUARAQUEÇABA (PR)
}

\author{
Maurício Bergamini Scheer* \\ *Eng. Florestal, M.Sc., Doutorando em Engenharia Florestal, UFPR, Companhia de Saneamento do Paraná, SANEPAR - \\ mauriciobs@sanepar.com.br
}

Recebido para publicação: 31/07/2007 - Aceito para publicação: 13/09/2007

\begin{abstract}
Resumo
Este trabalho tem o objetivo de avaliar o processo de decomposição da serapilheira foliar, analisando a perda de fitomassa, a liberação e a retenção de seus macronutrientes, micronutrientes e alumínio em um trecho de Floresta Ombrófila Densa Aluvial Atlântica em regeneração natural, durante o nono e décimo ano após o abandono da bubalinocultura, na Reserva Natural Salto Morato, em Guaraqueçaba, Paraná. Através da técnica das bolsas de decomposição "litter bags", foi estimado um tempo médio de um ano para que pelo menos a metade do material foliar depositado no solo da capoeira fosse decomposta. Como em outros estudos, a perda de material foi acentuada no início do processo (primeiros meses) e a taxa de decomposição foi inferior à de outras florestas tropicais, mas similar à de outros estudos na floresta atlântica. Houve uma tendência de aumento nos teores dos elementos analisados no material remanescente durante o processo de decomposição, exceto para o K e o C.

Palavras-chave: Ciclagem de nutrientes; serapilheira; decomposição; Floresta Aluvial Atlântica; sucessão secundária.
\end{abstract}

\begin{abstract}
Leaf litter decomposition and nutrient release on a secondary successional alluvial Atlantic Rain Forest in Southern Brazil. Leaf decomposition and nutrient release and retention were evaluated in an alluvial Atlantic Rain Forest in natural regeneration during the $9^{\text {th }}$ and $10^{\text {th }}$ years after Asian buffalo grazing was abandoned. The "litter bag" technique was used. At least half of the leaf litter disappeared in one year. Like other studies, weight loss was higher in the earlier stages (first months) of the decomposition process. Calculated decomposition rate was lower than in other tropical forests of the world, although similar to other Atlantic forests in Brazil. There was a tendency of increasing element concentration during the decomposition process, except for $\mathrm{K}$ and $\mathrm{C}$.

Keywords: Alluvial Atlantic Rain Forest; decomposition; leaf litter; nutrient release; secondary succession.
\end{abstract}

\section{INTRODUÇÃO}

As taxas de decomposição de serapilheira e de liberação de nutrientes no solo têm sido estudadas em uma ampla variedade de florestas, perfazendo muito mais que 1000 trabalhos já realizados no mundo, indicando a importância da decomposição na ciclagem de nutrientes dentro dos ecossistemas (GRUBB, 1995; PRESCOTT, 2005).

A maior parte da matéria orgânica acumulada na superfície do solo das florestas é constituída principalmente de material vegetal, transferido pela deposição de serapilheira. Dentre os componentes dessa manta orgânica, o material foliar é o mais significativo em quantidade de nutrientes orgânicos e inorgânicos (MEGURO et al., 1979). A decomposição depende do ambiente físico (temperatura, umidade, sazonalidade e fatores pedológicos), da composição do recurso (teores de lignina, celulose, compostos fenólicos, elementos minerais, substâncias estimulantes ou alelopáticas no material biológico) e conseqüentemente da comunidade de organismos decompositores (microflora e fauna) (EWEL, 1976; EDWARDS, 1977; MEGURO et al., 1980; TANNER, 1981; SWIFT; ANDERSON, 1989; PALM; 
SANCHEZ, 1990; ALVAREZ-SÁNCHEZ; BECERRA, 1996; GAMA; VILLELA, 2003; WISNIEWSKI et al., 1997; XULUC-TALOSA et al., 2003).

A decomposição da serapilheira resulta no acúmulo da matéria orgânica do solo, na liberação de seus nutrientes para a biota e na dissipação de parte do dióxido de carbono, sendo um processo que mantém a fertilidade e produtividade do sítio. É devido à eficiência do processo de ciclagem que ocorre nas camadas e horizontes mais superficiais do solo que a maior parte das florestas tropicais e subtropicais, como a maioria das formações da Floresta Ombrófila Densa, mantêm sua alta produtividade, mesmo em solos com baixa disponibilidade de nutrientes.

A vegetação nos estágios iniciais e intermediários de sucessão secundária que atualmente predomina, principalmente, nas áreas planas - como nas formações aluviais que foram degradadas na Floresta Ombrófila Densa (74\% de perda em relação à floresta original, de acordo com SEMA, 2002) tem estreita dependência da decomposição da serapilheira depositada pelas espécies pioneiras, as quais contribuem para a restituição da matéria orgânica dos horizontes superficiais do solo e para a disponibilização de nutrientes. Nesse processo, são criadas condições para a colonização por espécies vegetais mais exigentes, aumentando a produção primária (resiliência do sítio) e promovendo o avanço do processo sucessional.

Portanto, como parte de um projeto de pesquisa que visa contribuir para o entendimento de processos sucessionais num ambiente aluvial de Floresta Ombrófila Densa Atlântica, visando seu manejo e restauração, o presente estudo da ciclagem de nutrientes têm os objetivos de: (I) avaliar a decomposição foliar em um experimento com início no outono e em outro com início na primavera, e (II) avaliar a liberação e/ou retenção de elementos durante o processo de decomposição.

\section{MATERIAL E MÉTODOS}

\section{Área de estudo}

A área de estudo está situada em uma formação aluvial, a aproximadamente $20 \mathrm{~m}$ s.n.m., na Reserva Natural Salto Morato (RNSM), Guaraqueçaba, litoral norte do Paraná, no domínio da Floresta

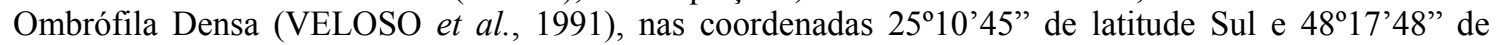
longitude Oeste (Figura 1). A RNSM é uma Reserva Particular do Patrimônio Natural da Fundação O Boticário de Proteção à Natureza que, em 1999, foi reconhecida como um Patrimônio Natural da Humanidade pela UNESCO. A planície aluvial é formada por sedimentos recentes (sedimentos inconsolidados - Cenozóico) originados da Serra do Morato, composta por granitos e migmatitos (FBPN, 1995; MINEROPAR, 2001). A área específica compreende sete hectares, onde a comunidade vegetal foi investigada por este estudo durante o nono e o décimo ano de regeneração após o abandono da bubalinocultura (1988-1993). Antes disso, a floresta havia sido derrubada e a área utilizada para cultivos agrícolas.

\section{Clima}

O clima na região, segundo Köppen, é Cfa, ou subtropical, com temperatura média no mês mais frio inferior a $18{ }^{\circ} \mathrm{C}$ (mesotérmico) e temperatura média no mês mais quente acima de $22^{\circ} \mathrm{C}$, com verões quentes, geadas pouco freqüentes e tendência de concentração das chuvas nos meses de verão, contudo sem estação seca definida. Os índices pluviométricos ultrapassam $2000 \mathrm{~mm}$ (IAPAR, 2005). No entanto, Maack (1968), apesar de encontrar valores levemente abaixo de $18{ }^{\circ} \mathrm{C}\left(\mathrm{de} 17\right.$ a $17,5{ }^{\circ} \mathrm{C}$ ) para a temperatura média do mês mais frio, em observações meteorológicas entre 30 e 60 anos, na estação meteorológica de Paranaguá, preferiu considerar o litoral como de clima tropical Af (acrescentando a letra t, por representar transição), por apresentar as mesmas condições fisiográficas do litoral paulista entre Santos e Cananéia. Através dos dados meteorológicos históricos de Guaraqueçaba disponibilizados pelo Instituto Agronômico do Paraná (IAPAR, 2006), foi elaborado o diagrama climático apresentado na figura 2. É possível notar basicamente a ausência de período seco e a predominância de um clima superúmido (precipitação $>100 \mathrm{~mm}$ ).

\section{Caracterização dos solos}

A área do estudo situa-se em uma planície aluvial, com uma distribuição de unidades pedológicas muito variável, formando um mosaico (FBPN, 1995). Segundo Mocochinski et al. (2003), em levantamento de solos no mesmo local, existe uma grande instabilidade das condições geomorfológicas, em função de pequenas inundações periódicas que podem ocorrer, geralmente entre dezembro e março. No trabalho desses autores, segundo o Sistema Brasileiro de Classificação de Solos 
(EMBRAPA, 1999), foi constatada a predominância de Neossolos Flúvicos (típico e gleico) e de Cambissolos Hísticos (Tabela 1), ocorrendo sob a vegetação com um maior grau de desenvolvimento, com dominância de árvores e arbustos. Já nos locais onde a sucessão vegetal parece ser muito mais lenta, com predomínio de plantas herbáceas, ocorrem preferencialmente Organossolos e Neossolos Litólicos (situação não estudada no presente trabalho).

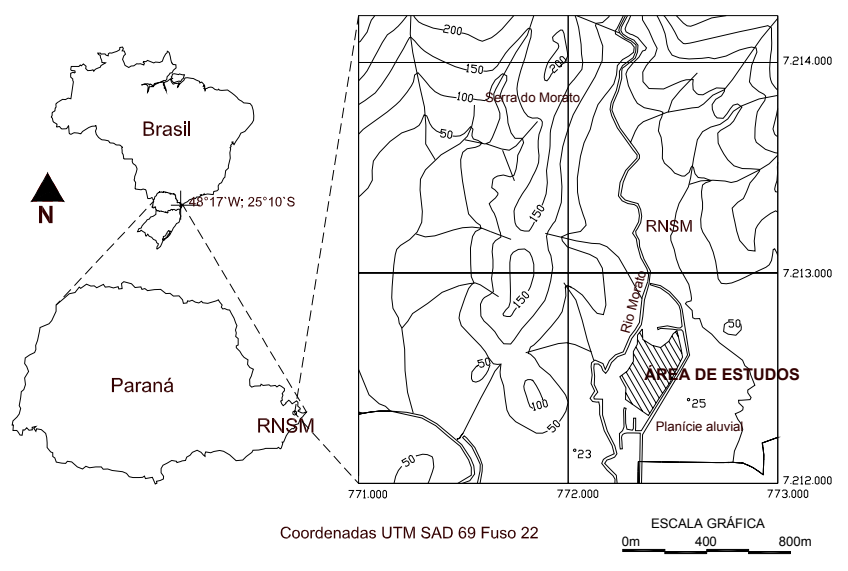

Figura 1. Mapa de localização geográfica da área de estudos (Reserva Natural Salto Morato) no município de Guaraqueçaba, PR.

Figure 1. Geographic location of the alluvial plain at Salto Morato Natural Reserve (RNSM), Guaraqueçaba, state of Paraná, Brazil.

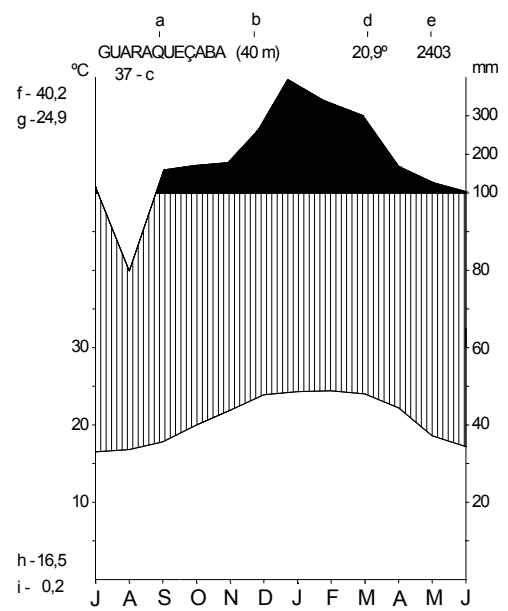

Figura 2. Diagrama climático construído segundo Walter (1986). Dados da estação meteorológica do IAPAR, em Guaraqueçaba (PR), período entre 1978 e 2005.

Figure 2. Climate diagram for Guaraqueçaba region, constructed according to Walter (1986). Data during the period from 1978 to 2005 (IAPAR, 2006).

Traço inferior $=$ curva de temperatura média; traço superior $=$ curva de precipitação pluviométrica; hachura vertical $=$ estação relativamente úmida; hachura escura= estação superúmida, precipitação média mensal $>100 \mathrm{~mm}$ (escala reduzida a 1/10); a= estação meteorológica; $\mathrm{b}=$ altitude; $\mathrm{c}=\mathrm{n}^{\mathrm{o}}$ de anos de observação; $\mathrm{d}=$ temperatura média anual; $\mathrm{e}=$ precipitação média anual; $\mathrm{f}=$ temperatura máxima absoluta; $\mathrm{g}=$ temperatura média diária do mês mais quente; $\mathrm{h}=$ temperatura média diária do mês mais frio; $\mathrm{i}=$ temperatura mínima absoluta.

*botton line $=$ mean temperature; upper line $=$ rainfall; vertical lines hatchet area $=$ wet season; black area $=$ monthly rainfall $\geq 100$ $\mathrm{mm}$ (scale reduced by $1 / 10$ ); $\mathrm{a}=$ location of meteorological station; $\mathrm{b}=$ altitude; $\mathrm{c}=$ length of observation period; $\mathrm{d}=$ annual average of temperature; $\mathrm{e}=$ mean annual rainfall; $\mathrm{f}=$ absolute maximum temperature; $\mathrm{g}=$ mean temperature of the warmest month; = mean temperature of the coldest month; $\mathrm{i}=$ absolute mean temperature. 
Tabela 1. Média de resultados $(\mathrm{n}=10)$ de análises químicas dos solos sob a capoeira.

Table 1. Mean $(\mathrm{n}=10)$ values for top soil chemical properties for the capoeira site (Cambisols and Fluvisols).

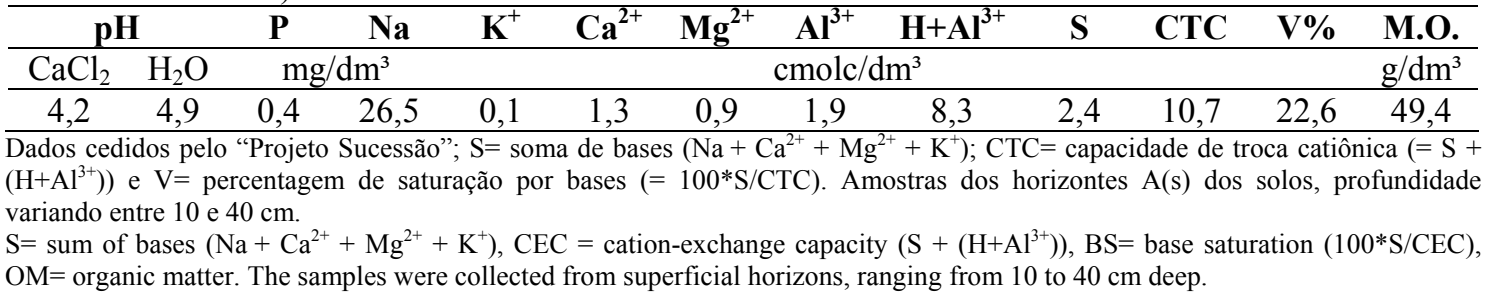

\section{Caracterização da vegetação}

Segundo Gatti (2000), apesar da mesma idade de regeneração (dez anos), na área estudada existem ambientes em diferentes velocidades de regeneração, influenciados provavelmente pela morfologia dos solos, sua saturação hídrica e nível de degradação, e também pela presença de densos agrupamentos de espécies exóticas invasoras com alto potencial competitivo, como Brachiaria spp.

De acordo com a classificação de sucessão secundária proposta por Klein (1980), a vegetação pode ser enquadrada como "capoeira". Essa área localiza-se a aproximadamente $500 \mathrm{~m}$ de uma floresta secundária submontana avançada, fonte de propágulos. No levantamento fitossociológico realizado (dados não publicados), foram encontradas 36 espécies lenhosas, apresentando uma densidade de 2820 indivíduos/há, uma área basal de $22,2 \mathrm{~m}^{2} /$ há e perímetro à altura do peito $(\mathrm{PAP})>10 \mathrm{~cm}$. Nessa vegetação predominam indivíduos arbóreos com altura média de oito metros, ocorrendo Myrsine coriacea (Sw.) R. Br. Ex Roem. \& Schult., Senna multijuga (Rich.) H. S. Irwin \& Barneby, Cecropia pachystachya Trécul, Tibouchina pulchra Cogn., Psidium guajava L. (exótica) e remanescentes de Citharexylum myrianthum Cham., freqüentemente formando um dossel contínuo. Entre as espécies arbustivas são comuns Piper aduncum L., Boehmeria caudata Sw. e Solanum fastigiatum Willd. As principais espécies herbáceas ocorrentes na área são: Vernonia scorpioides (Lam.) Pears., Manettia congesta (Vell.) K. Schum., Rubus rosifolius Stokes var. rosifolius e Clidemia hirta D. Don, entre outras. Também ocorrem indivíduos jovens de espécies arbóreas que provavelmente irão dominar a comunidade no próximo estágio sucessional, como Hyeronima alchorneoides M. Allem., Vochysia bifalcata Warm., Matayba sp., Cordia sylvestris Sw., Euterpe edulis e Hirtella hebeclada Moric. ex DC., entre outras.

\section{Decomposição foliar e liberação de nutrientes}

Para estudar a decomposição da serapilheira foliar, foi usada uma adaptação da técnica das bolsas de decomposição "nylon hairnets" ou "litter bags", descrita por Bocock; Gilbert, 1957.

Foram realizados dois experimentos. O primeiro (experimento 1) teve início no outono (em maio de 2002, início do período menos chuvoso) e o segundo (experimento 2) teve início na primavera (em novembro de 2002, início do período mais chuvoso e quente - Figura 2). Para cada experimento, o material foliar recém-caído e em fase terminal de abscisão foi coletado na capoeira, durante dois dias, na mesma semana do início de cada experimento. O material foi previamente separado em grupos de folhas das espécies mais importantes na estrutura arbórea da capoeira, sendo: Myrsine coriacea, Alchornea glandulosa, Psidium guajava, Senna multijuga, Cecropia pachystachya, Tibouchina pulchra e Citharexylum myrianthum. Parte do material foi destinada à composição de 60 bolsas de náilon, com malha de $2 \mathrm{~mm}$ e dimensões de $20 \times 20 \mathrm{~cm}$, contendo uma proporção de cada fração do material disponível (média ponderada equivalente à serapilheira depositada), de modo a reduzir a variabilidade dos resultados. Para cada experimento, a proporção das espécies foi considerada equivalente à da produção de serapilheira no período. O material de cada bolsa foi pesado na umidade ambiente, correspondendo ao tempo inicial de decomposição, somando aproximadamente 20 gramas cada. A outra parte do material coletado foi destinada a uma calibragem para se obter uma relação entre a fitomassa seca e a fitomassa na umidade ambiente de amostras similares ao conteúdo das bolsas. Tal procedimento permitiu a obtenção de uma estimativa da massa seca inicial do material do experimento sem interferir com a secagem artificial dessas amostras. Edwards (1977) e Tanner (1981) comentam que a secagem do material antes dos experimentos de decomposição pode resultar em uma taxa de decomposição mais lenta. Um conjunto 
de amostras foi destinado às análises químicas, para se obterem as concentrações iniciais de macro e micronutrientes e alumínio do material recém-caído.

As bolsas, devidamente identificadas, foram distribuídas sobre a superfície do solo da capoeira em linhas de 10 unidades. Nos intervalos de 0,5, 1, 3, 6, 9 e 12 meses após sua colocação, o material foi recolhido cuidadosamente, retirando-se partículas de solo aderidas e/ou raízes que se infiltraram nas bolsas. Foram retiradas 10 bolsas por coleta. As amostras foram enviadas ao laboratório para a pesagem (massa seca), e, para diminuir custos, somente as amostras do experimento1 foram submetidas à análise química para a determinação dos teores dos elementos minerais (agrupando-se em três repetições). Foram estimadas as taxas instantâneas de decomposição (coeficiente $\mathrm{K}$ ) e os tempos necessários para a decomposição de 50 e $95 \%$ do material, através do modelo exponencial simples utilizado por Jenny et al. (1949) e Olson (1963). Wieder; Lang (1982) afirmam que, geralmente, os modelos exponenciais são mais realísticos em termos de comportamento matemático e biológico. Segundo Prescott (2005), o modelo exponencial é limitado aos estudos de curta duração, entre um e dois anos. Em investigações mais longas, após determinado tempo, a decomposição alcança um limite que corresponde à transformação da serapilheira em húmus, sendo uma nova fase do processo (ABER; MELLILO, citados por PRESCOTT, 2005). Também foram estimadas as relações $\mathrm{C} / \mathrm{N}$ e as taxas de liberação ou retenção dos nutrientes com o processo de decomposição.

Para o cálculo do coeficiente de decomposição $(\mathrm{K})$ foi utilizada a seguinte fórmula matemática:

$\mathrm{K}=-\ln \left(1-\mathrm{K}^{\prime}\right)$, onde $\mathrm{K}$ ' é a razão entre a fitomassa perdida em determinado período e a fitomassa inicial.

Para o cálculo do tempo necessário para decompor 50 e 95\% do material:

$$
\begin{gathered}
\mathrm{T}_{50 \%}=0,693 / \mathrm{K} \\
\mathrm{T}_{95 \%}=3 / \mathrm{K}
\end{gathered}
$$

\section{Análises químicas}

Todas as análises químicas foram efetuadas no Laboratório de Biogeoquímica e Nutrição Mineral de Plantas (LABINP) do Departamento de Solos e Engenharia Agrícola da Universidade Federal do Paraná, exceto as análises de carbono, que foram realizadas pela equipe do Laboratório de Biogeoquímica Ambiental do Centro de Energia Nuclear na Agricultura (CENA/USP).

$\mathrm{O} \mathrm{N}$ nas amostras vegetais foi determinado pelo método Kjeldhal, descrito por Hildebrand (1977). Esse método consiste basicamente em uma digestão úmida com ácido sulfúrico concentrado, seguida de uma destilação com hidróxido de sódio (32\%) e titulação ácida do amônio. A determinação dos demais elementos foi realizada digerindo-se o material em mufla a $500{ }^{\circ} \mathrm{C}$ e solubilizando-o em $\mathrm{HCl}$ $10 \%$, conforme descrito por Perkin-Elmer Corporation (1973). As leituras de $\mathrm{K}, \mathrm{Ca}, \mathrm{Mg}, \mathrm{Fe}, \mathrm{Mn}, \mathrm{Cu}, \mathrm{Zn}$ e Al foram realizadas em espectrofotômetro de absorção atômica 2380 Perkin-Elmer (K por emissão). O $\mathrm{P}$ foi determinado pelo método colorimétrico com vanadato molibdato de amônio (cor amarela), com leitura em espectrofotômetro UV/VIS 554 Perkin-Elmer. As análises de carbono foram realizadas pelo método de via seca por combustão no aparelho determinador LECO CN 2000. O mesmo aparelho também determinou teores de $\mathrm{N}$ para as mesmas amostras.

\section{Análises estatísticas}

Foram calculados os erros padrões para as perdas de massa seca e dos elementos químicos nos diferentes tempos de decomposição. Para verificar diferenças estatísticas na decomposição de fitomassa e nos teores de nutrientes entre os períodos amostrados, foram realizados testes de comparação de médias (testes de $\mathrm{t}$ de Student e de Tukey, $\mathrm{p}<0,05$ ). Os dados foram transformados em log de $\mathrm{x}$, para atender requisitos de normalidade, homogeneidade de variâncias e aditividade. Foram utilizados os coeficientes de correlação de Spearman $\left(\mathrm{r}_{\mathrm{s}}\right)$ para análises não-paramétricas.

\section{RESULTADOS E DISCUSSÃO}

\section{Decomposição foliar}

Nas tabelas 2 e 3, estão apresentados os percentuais médios de decomposição do material foliar com diferentes períodos de permanência no piso florestal. Após um ano do experimento 1, com início no outono, aproximadamente $50 \%$ do material foi decomposto. No experimento 2 , com início na primavera, 
após um ano, aproximadamente $62 \%$ do material foliar foi decomposto, mas não foram encontradas diferenças estatisticamente significativas entre os dois experimentos após 12 meses de decomposição (teste de $\mathrm{t}, \mathrm{n}=10$ ). Esses valores de decomposição estão dentro da faixa (em torno dos 55\%) encontrada por Varjabedian; Pagano (1988), em um trecho de Floresta Ombrófila Densa Submontana, e por Wisniewski et al. (1997) e Rocha et al. (2003), em trechos de Floresta Ombrófila Densa das Terras Baixas. As estimativas do tempo necessário para a redução de $50 \%$ do peso do material em decomposição foram de 1,0 e 0,7 anos, respectivamente, para os experimentos com início no outono e com início em novembro. Para a decomposição de 95\% do material, as estimativas foram de 4,4 e 3,2 anos. Os valores do experimento 2, foram similares aos registrados por Santos (1989) em Floresta Estacional Semidecidual no Paraná.

Os valores de 0,68 e 0,95 para o índice K (tabelas 2 e 3) estão próximos (em torno de 0,75) aos encontrados por Varjabedian; Pagano (1988), De Vuono et al. (1989), Wisniewski et al. (1997) e Portes (2001), em floresta atlântica no Sudeste e Sul do Brasil, sendo esses valores considerados baixos para florestas tropicais.

As folhas das espécies utilizadas nas bolsas do presente estudo, como $C$. pachystachya, $P$. guajava e $C$. myrianthum apresentam texturas coriáceas, com pecíolos grandes e muitas nervuras salientes, de difícil decomposição. Heath e Arnold, citados por Meguro et al. (1980), afirmaram que espécies heliófilas, com textura rígida, apresentam geralmente maior dificuldade de umedecimento quando mortas, o que desfavorece a atividade de microorganismos. Edwards (1977), Varjabedian; Pagano (1988) e Swift; Anderson (1989) relataram que baixos valores de $\mathrm{pH}$, alta acidez potencial $\left(\mathrm{H}^{+}+\mathrm{Al}^{+}\right)$e excesso de umidade e de matéria orgânica do solo retardam a atividade de organismos, diminuindo a velocidade de decomposição. Conforme a Tabela 1, os solos da capoeira estudada apresentam um $\mathrm{pH}$ próximo a 4,5, sendo classificados como fortemente ácidos (EMBRAPA, 1999).

$\mathrm{Na}$ figura 3, estão representados graficamente os percentuais de material foliar remanescente sobre o solo para os dois experimentos. É possível notar um mesmo padrão nas duas curvas de decomposição, também encontrado por Oliveira (1987) e Portes (2001), no qual a perda de material é acentuada no primeiro trimestre e no segundo há uma diminuição na velocidade de decomposição, independentemente da época do ano. Nas fases iniciais da decomposição, ocorre a fragmentação de partículas por agentes físicos e pela biota e a liberação de compostos mais solúveis, como açúcares, amido e proteínas, os quais são rapidamente utilizados pelos decompositores. Após esse período, grande parte das estruturas mais resistentes, ricas em lignina, celulose, gorduras, ceras e taninos, como nervuras e pecíolos, ainda permanece, diminuindo a velocidade de decomposição (EWEL, 1976; MEGURO, 1980; WIEDER; LANG, 1982).

Diferenças estatisticamente significativas entre os experimentos só foram observadas para o material com 15 dias de decomposição, indicando a maior decomposição inicial no período mais quente e úmido (experimento 2) (teste de $\mathrm{t}, \mathrm{n}=10$ ). Luizão; Schubart (1986), entre outros autores, atribuem a maior velocidade de decomposição na estação chuvosa às condições de umidade, que favorecem a atividade de organismos decompositores, principalmente artrópodes que removem a liteira e que também estimulam o crescimento das raízes superficiais, que penetram no material em decomposição.

Tabela 2. Percentual médio de decomposição do material foliar em cada período de coleta (experimento 1 , início no outono); $\mathrm{K}=$ razão instantânea de decomposição; $\mathrm{n}=10$.

Table 2. Mean leaf litter loss (\%) during decomposition process (experiment 1); $\mathrm{K}=$ decomposition rate; $\mathrm{n}=10$.

\begin{tabular}{lcccc}
\hline $\begin{array}{l}\text { Período } \\
\text { (meses) }\end{array}$ & Data da coleta & K & \% decomposta & Erro padrão \\
\hline 0 & $22 / 05 / 02$ & 0 & 0,0 & 0,0 \\
0,5 & $08 / 06 / 02$ & 0,06 & $6,4 \mathrm{a}$ & 2,2 \\
1 & $22 / 06 / 02$ & 0,13 & $12,1 \mathrm{ab}$ & 3,6 \\
3 & $22 / 08 / 02$ & 0,33 & $28,2 \mathrm{bc}$ & 4,9 \\
6 & $22 / 11 / 02$ & 0,37 & $30,9 \mathrm{~cd}$ & 2,1 \\
9 & $22 / 02 / 03$ & 0,60 & $44,9 \mathrm{~cd}$ & 2,9 \\
12 & $18 / 05 / 03$ & 0,68 & $49,6 \mathrm{~d}$ & 4,6 \\
\hline
\end{tabular}

Médias seguidas de letras iguais não diferem entre si pelo teste de Tukey; $\mathrm{p}<0,05$. 
Tabela 3. Percentual médio de decomposição do material foliar em cada período de coleta (experimento 2, início na primavera); $\mathrm{K}=$ razão instantânea de decomposição; $\mathrm{n}=10$.

Table 3. Mean leaf litter loss (\%) during decomposition process (experiment 2); $\mathrm{K}=$ decomposition rate; $n=10$.

\begin{tabular}{lcccc}
\hline $\begin{array}{l}\text { Período } \\
\text { (meses) }\end{array}$ & Data da coleta & K & \% decomposta & Erro padrão \\
\hline 0 & $24 / 11 / 02$ & 0 & 0,0 & 0,0 \\
0,5 & $14 / 12 / 02$ & 0,14 & $12,9 \mathrm{a}$ & 2,1 \\
1 & $27 / 12 / 02$ & 0,18 & $16,7 \mathrm{a}$ & 2,3 \\
3 & $22 / 02 / 03$ & 0,29 & $25,2 \mathrm{ab}$ & 3,3 \\
6 & $18 / 05 / 03$ & 0,38 & $31,6 \mathrm{bc}$ & 3,9 \\
9 & $24 / 08 / 03$ & 0,76 & $53,1 \mathrm{c}$ & 2,8 \\
12 & $24 / 11 / 03$ & 0,95 & $61,5 \mathrm{c}$ & 4,3 \\
\hline
\end{tabular}

Médias seguidas de letras iguais não diferem entre si pelo teste de Tukey; $\mathrm{p}<0,05$.

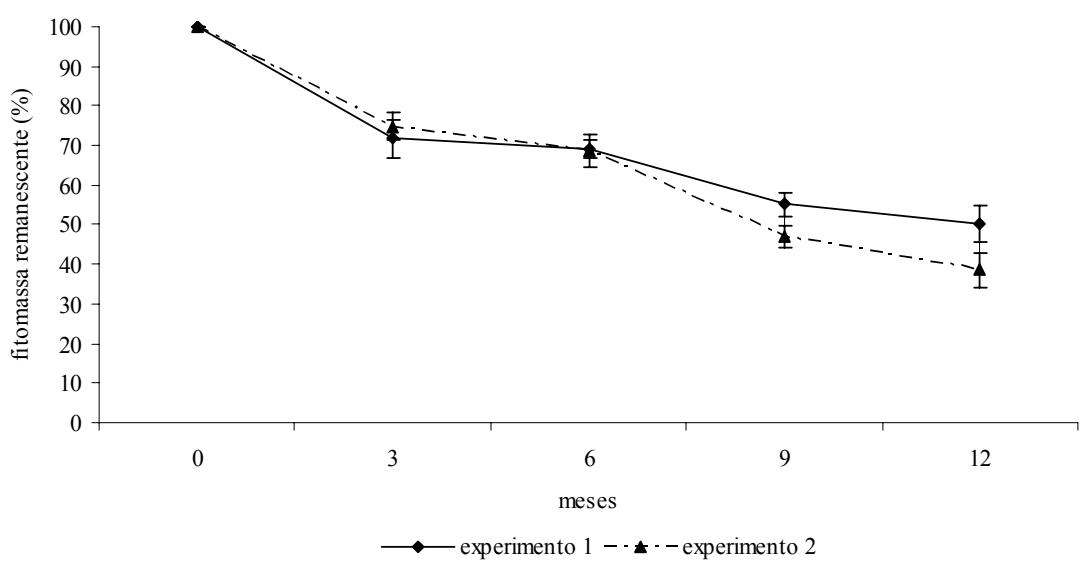

Figura 3. Percentuais médios do peso remanescente da serapilheira foliar em decomposição nos experimentos 1 e 2 e respectivos erros padrões.

Figure 3. Mean leaf litter remnant (\%) in litter bags during decomposition process.

\section{Teores de nutrientes do material em decomposição}

Liberação de carbono do material foliar

Com base na tabela 4, os teores de C mantiveram-se em torno de $440 \mathrm{~g} / \mathrm{kg}$ até os seis meses de decomposição. Entre os seis e 12 meses, o valor diminuiu para aproximadamente $340 \mathrm{~g} / \mathrm{kg}$. Já o conteúdo (quantidade) de C remanescente diminuiu, acompanhando a perda de fitomassa, restando aproximadamente 39\% (Tabela 6 e Figura 4). Portanto, aproximadamente $61 \%$ do $\mathrm{C}$ deixou o material foliar e foi incorporado nas camadas dos horizontes superficiais do solo (incluído na sua biota) e/ou dissipado para a atmosfera. Segundo Scheer (2006), essa vegetação depositou 6,4 t/ha/ano de serapilheira. Levando em consideração um teor inicial de C nesse material de cerca de 44\% (Tabela 4), é possível estimar uma transferência no ano estudado de aproximadamente 2,8 t/ha de $\mathrm{C}$ para o piso florestal, podendo $61 \%(1,7 \mathrm{t})$ serem liberados em 1 ano para outros compartimentos do ecossistema. A liberação desse elemento foi fortemente correlacionada com a perda de massa seca $\left(r_{s}=1\right)$, com a liberação de $\mathrm{K}$ e $\mathrm{Mg}$ e com a retenção de $\mathrm{Fe}$ e $\mathrm{Al}\left(\mathrm{r}_{\mathrm{s}}=0,86, \mathrm{p}<0,04, \mathrm{n}=7\right)$.

\section{Liberação de macronutrientes do material foliar}

As concentrações de $\mathrm{N}$ aparentemente indicaram um aumento relacionado com o tempo de decomposição. Apenas os teores iniciais diferiram estatisticamente dos teores do material após um ano de decomposição (Tukey, $\mathrm{n}=3$ ). $\mathrm{O}$ aumento dos teores de $\mathrm{N}$ pode ser atribuído, além da perda mais rápida de $\mathrm{C}$ e de massa seca, à adição via precipitações atmosféricas, à fixação simbiótica e assimbiótica (pela 
colonização e utilização pela biota decompositora) e por contaminação do material via queda de materiais de origem animal e vegetal (THOMPSON; VITOUSEK, 1997). Segundo Luizão (1982), a presença de proteínas nos microdecompositores que morrem e são analisados junto com as folhas em decomposição e de outros compostos orgânicos resultantes da ação da biota produz um aumento considerável nos teores de $\mathrm{N}$ e de enxofre no material. Como no trabalho de Edwards (1977), muitas bolsas foram recolhidas contendo micélios de fungos. De acordo com a tabela 6 e a figura $4,71,5 \%$ de $\mathrm{N}$ do conteúdo inicial ainda permaneciam nas bolsas, diferindo um pouco dos resultados obtidos em florestas estacionais por Meguro et al. (1980) e por Santos (1989), respectivamente, 65 e 44\%. Foi notado um decréscimo na relação C/N e nos teores de K ao longo do tempo de decomposição. Myers et al., citado por Arunachalam et al. (1998), relataram que substratos com a relação $\mathrm{C} / \mathrm{N}<25$ têm uma maior taxa de liberação de $\mathrm{N}$. Isso só ocorreu entre o primeiro e o terceiro mês de decomposição em diante (Tabela 4). A partir disso, o $\mathrm{N}$ começou a ser liberado (diminuiu o conteúdo do elemento no material remanescente), conforme consta na figura 4. Thompson; Vitousek (1997), em experimento no Havaí, detectaram um aumento nos teores de N até 70 dias, sendo que a partir disso os teores foram diminuindo. Nesse mesmo trabalho, os autores observaram que a maior imobilização desse elemento e também do P estava relacionada com a menor disponibilidade desses elementos no solo. $\mathrm{O}$ comportamento do $\mathrm{N}$ durante a decomposição foi fortemente correlacionado com o do $\mathrm{Mg}\left(\mathrm{r}_{\mathrm{s}}=0,89 ; \mathrm{p}<0,03\right)$.

Tabela 4. Teores médios e erros padrões de macronutrientes e de carbono no material vegetal com diferentes tempos de decomposição na capoeira (experimento 1); em g/kg; $\mathrm{n}=3$.

Table 4. Carbon and macronutrient mean concentrations in leaf litter during decomposition process. The standard errors are between parentheses; $\mathrm{g}_{\mathrm{kg}} \mathrm{kg}^{-1} ; \mathrm{n}=3$.

\begin{tabular}{|c|c|c|c|c|c|c|c|c|}
\hline $\begin{array}{l}\begin{array}{l}\text { Período } \\
\text { (meses) }\end{array}\end{array}$ & C & $\mathbf{N}$ & $\mathbf{C} / \mathbf{N}^{*}$ & $\mathbf{N}$ & $\mathbf{P}$ & $\mathbf{K}$ & Ca & Mg \\
\hline 0 & 440,8 & 13,8 & 31,9 & $\begin{array}{lll}13,0 & (1,7)\end{array}$ & $\begin{array}{ll}0,9 & (0,12)\end{array}$ & $5,2 \quad(0,3)$ & $12,4 \quad(1,5)$ & $\begin{array}{ll}2,9 & (0,3)\end{array}$ \\
\hline 0,5 & 437,6 & 13,7 & 31,8 & $17,3 \quad(0,6)$ & $1,1 \quad(0,10)$ & $4,0 \quad(0,2)$ & $13,1 \quad(1,3)$ & $3,0 \quad(0,3)$ \\
\hline 1 & 446,0 & 15,6 & 28,6 & $16,9 \quad(2,3)$ & $1,2 \quad(0,08)$ & $2,6 \quad(0,2)$ & $14,8 \quad(0,8)$ & $3,4 \quad(0,1)$ \\
\hline 3 & 450,1 & 22,1 & 20,4 & $18,4 \quad(1,8)$ & $1,6 \quad(0,02)$ & $1,5 \quad(0,1)$ & $17,2 \quad(0,8)$ & $3,4 \quad(0,2)$ \\
\hline 6 & 451,0 & 19,0 & 23,7 & $14,7 \quad(1,7)$ & $1,6 \quad(0,02)$ & $1,2 \quad(0,1)$ & $18,9 \quad(1,2)$ & $3,1 \quad(0,3)$ \\
\hline 9 & 373,7 & 15,4 & 24,3 & $19,5 \quad(0,7)$ & $1,6 \quad(0,04)$ & $1,5 \quad(0,1)$ & $22,0 \quad(2,9)$ & $3,1 \quad(0,2)$ \\
\hline 12 & 339,3 & 17,0 & 20,0 & $18,5 \quad(0,4)$ & $1,7 \quad(0,08)$ & $2,1 \quad(0,2)$ & $17,0 \quad(1,1)$ & $3,0 \quad(0,4)$ \\
\hline
\end{tabular}

Com base nos teores de $\mathrm{P}$ apresentados na tabela 4, é possível notar um aumento nos três primeiros meses, chegando a duplicar seu teor após um ano de exposição, fazendo com que a taxa de perda de fitomassa fosse amortizada pela taxa de ganho de teor desse elemento, resultando numa perda de conteúdo insignificante após um ano de decomposição (Figura 4). Como para o N, nesse aumento está envolvida a atividade de microorganismos. Somente após três meses de decomposição a relação N/P ficou abaixo de 10, sugerida como razão a partir da qual a decomposição microbiana é favorecida (VOGT et al., 1986).

$\mathrm{O}$ teor de $\mathrm{K}$ sofreu uma diminuição de $71 \%$ nos três primeiros meses, de 5,2 para $1,5 \mathrm{~g} / \mathrm{kg}$ da massa seca, terminando o período dos 12 meses com um teor de $2,1 \mathrm{~g} / \mathrm{kg}$ (Tabela 4). Após três meses, aproximadamente $80 \%$ do $\mathrm{K}$ haviam sido liberado do confinamento das bolsas (Tabela 5). Meguro et al. (1980) encontraram valores de perda desse elemento entre 30 e 90\%, e Lisanework; Michelsen (1994), entre 32 e $49 \%$ em três meses. Por esse elemento não estar fortemente associado à estrutura das folhas, ele é facilmente lixiviável. O pequeno aumento no conteúdo após um ano, encontrado por Meguro et al. (1980) e Luizão (1982), também se deve à atividade de microorganismos no folhedo, ou à adsorção desse elemento depositado pela atmosfera, ou apenas ao erro experimental.

Para o $\mathrm{Ca}$, houve também um aumento nos teores, demonstrando um padrão de retenção nos três primeiros meses. Como para o $\mathrm{P}$, a imobilização do Ca se estendeu até o sexto mês, quando seus conteúdos apresentaram uma sensível queda para $69 \%$ do conteúdo inicial. Segundo Santos (1989), o aumento nos teores de Ca se deve à presença desse elemento nas partes estruturais das folhas, que são de lenta decomposição. Segundo Cromack et al., citados por De Vuono et al. (1989), o aumento relativo de Ca no material remanescente pode também ser atribuído à absorção acentuada do elemento por hifas de fungos. 
Tabela 5. Percentual médio remanescente de carbono e de macronutrientes em relação ao conteúdo inicial do material foliar submetido a diferentes tempos de decomposição (experimento 1).

Table 5. Mean percentages of carbon and macronutrient in leaf litter remnant during decomposition process in relation to initial contents.

\begin{tabular}{lcccccc}
\hline $\begin{array}{l}\text { Período } \\
\text { (meses) }\end{array}$ & $\mathbf{C}$ & $\mathbf{N}$ & $\mathbf{P}$ & $\mathbf{K}$ & $\mathbf{C a}$ & $\mathbf{M g}$ \\
\hline 0 & 100,0 & 100,0 & 100,0 & 100,0 & 100,0 & 100,0 \\
0,5 & 96,0 & 128,2 & 124,1 & 74,4 & 102,6 & 100,2 \\
1 & 88,9 & 114,4 & 122,4 & 44,3 & 105,1 & 103,5 \\
3 & 73,3 & 101,7 & 131,0 & 20,7 & 99,9 & 83,1 \\
6 & 70,7 & 78,3 & 129,3 & 15,5 & 105,7 & 74,6 \\
9 & 46,7 & 82,5 & 102,2 & 15,7 & 97,7 & 58,5 \\
12 & 38,8 & 71,5 & 98,7 & 20,1 & 69,2 & 52,7 \\
\hline
\end{tabular}

Após um ano de decomposição, aproximadamente $47 \%$ do Mg foi liberado, apresentando uma taxa similar a da perda de carbono e da massa seca (Tabela 6, Figura 4). Como já mencionado, esse elemento também foi correlacionado positivamente com o comportamento do conteúdo de $\mathrm{N}$ durante a decomposição.

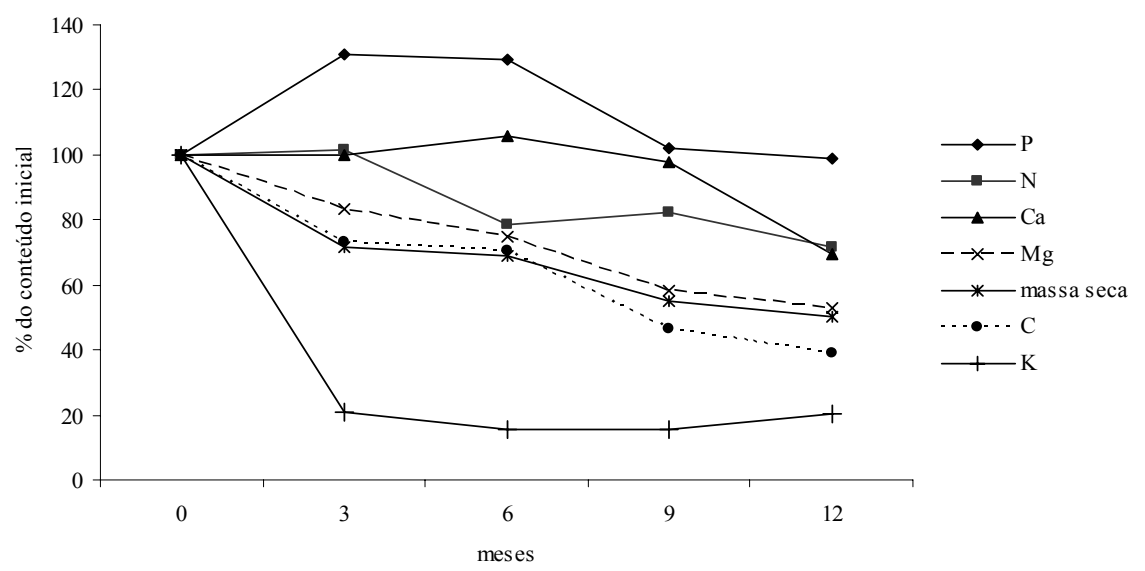

Figura 4. Percentuais médios de massa seca, carbono e de macronutrientes em relação ao conteúdo inicial do material foliar submetido a diferentes tempos de decomposição (experimento 1).

Figure 4. Mean percentages of dry mass, $\mathrm{C}$ and macronutrients during decomposition process in relation to initial contents.

\section{Liberação de aluminio e micronutrientes do material foliar}

Nos resultados das análises de $\mathrm{Cu}, \mathrm{Mn}, \mathrm{Fe}, \mathrm{Zn}$ e $\mathrm{Al}$ houve um aumento dos teores ao longo da decomposição, fato também encontrado por Sampaio et al. (1988) e De Vuono et al. (1989), entre outros autores (Tabela 5). As concentrações de $\mathrm{Cu}, \mathrm{Mn}$ e $\mathrm{Zn}$ duplicaram, enquanto que as de $\mathrm{Fe}$ e de $\mathrm{Al}$ aumentaram, respectivamente, 20 e 8 vezes após um ano. Já os conteúdos de Fe e Al aumentaram 10 e 4 vezes, respectivamente (Tabela 7 e Figura 5). Foi observada uma forte correlação entre os conteúdos desses dois elementos durante a decomposição $\left(r_{s}=0,86 ; p<0,04\right)$. Schubart et al. (1984) e Santos (1989) justificaram esse aumento pela contaminação do material confinado por partículas do solo, transportadas pela fauna e pelo desenvolvimento de raízes. Tal transporte pode também ocorrer pelo impacto das gotas da chuva. As raízes das plantas podem, por exemplo, estar acumulando Al na rizosfera (contaminando o conteúdo das bolsas em decomposição), evitando o deslocamento de quantidades tóxicas desse elemento para suas partes superiores (EPSTEIN, citado por LUIZÃO; SCHUBART, 1986). Os demais elementos apresentaram aumento nos seus teores, provavelmente devido à degradação mais rápida da massa seca, principalmente do $\mathrm{C}$. 
Tabela 6. Teores médios e erros padrões dos micronutrientes e de alumínio no material vegetal com diferentes tempos de decomposição na capoeira (experimento 1); em $\mathrm{mg} / \mathrm{kg} ; \mathrm{n}=3$.

Table 6. Aluminium and micronutrient mean concentrations in leaf litter during decomposition process. The standard errors are between parentheses; $\mathrm{mg} \cdot \mathrm{kg}^{-1} ; \mathrm{n}=3$.

\begin{tabular}{lllllllllll}
\hline $\begin{array}{l}\text { Período } \\
\text { (meses) }\end{array}$ & \multicolumn{2}{c}{ Fe } & \multicolumn{2}{c}{ Mn } & \multicolumn{2}{c}{ Cu } & \multicolumn{2}{c}{ Zn } & \multicolumn{2}{l}{ Al } \\
\hline 0 & 125,9 & $(9,5)$ & 155,1 & $(19,4)$ & 6,3 & $(1,2)$ & 13,5 & $(1,4)$ & 810,2 & $(370,4)$ \\
0,5 & 154,7 & $(14,3)$ & 165,2 & $(12,8)$ & 6,3 & $(2,0)$ & 13,5 & $(1,2)$ & 2485,7 & $(929,4)$ \\
1 & 160,4 & $(11,7)$ & 189,7 & $(5,7)$ & 10,3 & $(1,2)$ & 16,5 & $(0,8)$ & 1061,4 & $(52,4)$ \\
3 & 191,6 & $(18,3)$ & 194,9 & $(19,0)$ & 11,3 & $(3,0)$ & 19,2 & $(1,4)$ & 1034,2 & $(95,1)$ \\
6 & 388,7 & $(30,5)$ & 219,5 & $(9,3)$ & 8,9 & $(1,0)$ & 22,4 & $(0,7)$ & 2338,9 & $(492,6)$ \\
9 & 2138,2 & $(727,6)$ & 305,8 & $(13,5)$ & 14,9 & $(1,2)$ & 26,8 & $(1,1)$ & 4026,2 & $(896,3)$ \\
12 & 2610,4 & $(769,3)$ & 322,2 & $(32,7)$ & 12,1 & $(0,3)$ & 25,4 & $(1,8)$ & 6681,2 & $(1558,3)$ \\
\hline
\end{tabular}

Tabela 7. Percentual médio remanescente de micronutrientes e de alumínio em relação ao conteúdo inicial do material foliar submetido a diferentes tempos de decomposição (experimento 1).

Table 7. Mean percentages of aluminium and micronutrient in leaf litter remnant during decomposition process in relation to initial contents.

\begin{tabular}{lccccc}
\hline $\begin{array}{l}\text { Período } \\
\text { (meses) }\end{array}$ & $\mathbf{F e}$ & $\mathbf{M n}$ & $\mathbf{C u}$ & $\mathbf{Z n}$ & $\mathbf{A l}$ \\
\hline 0 & 100,0 & 100,0 & 100,0 & 100,0 & 100,0 \\
0,5 & 118,8 & 103,0 & 97,0 & 96,8 & 296,7 \\
1 & 112,0 & 107,5 & 144,3 & 107,8 & 115,2 \\
3 & 109,3 & 90,2 & 129,7 & 102,4 & 91,6 \\
6 & 213,4 & 97,8 & 98,3 & 114,7 & 199,5 \\
9 & 936,2 & 108,7 & 131,1 & 109,4 & 273,8 \\
12 & 1045,4 & 104,7 & 97,5 & 95,1 & 415,6 \\
\hline
\end{tabular}

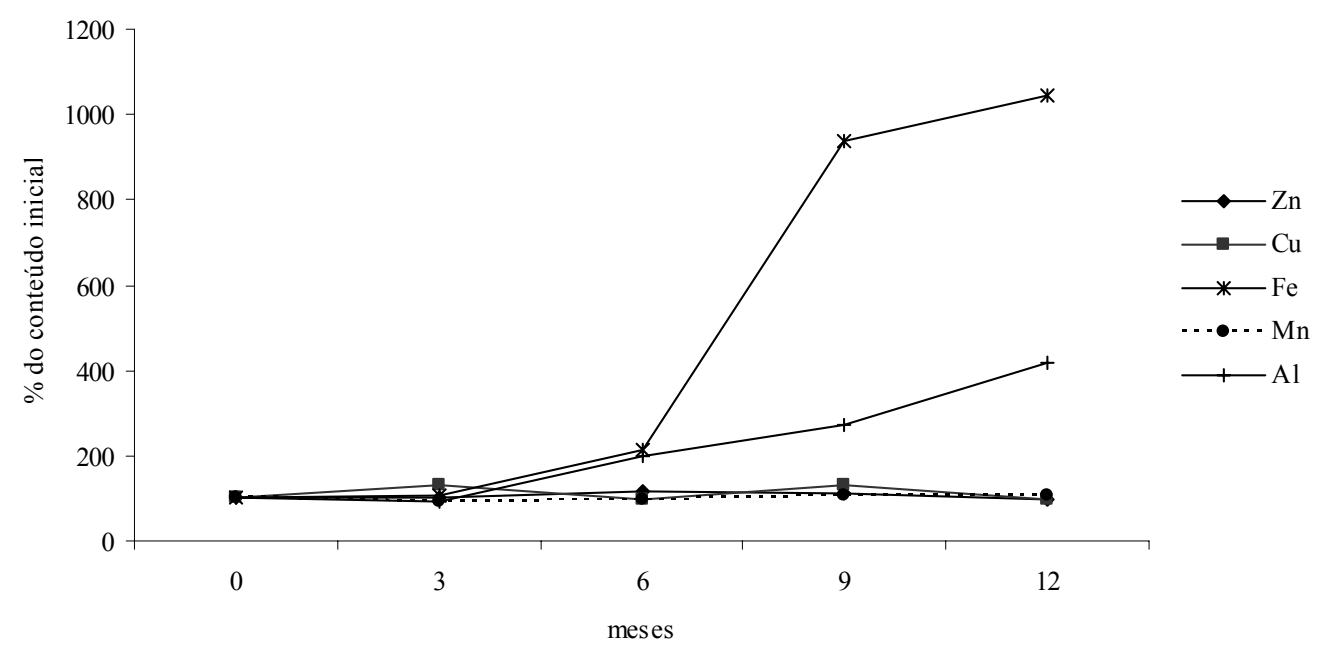

Figura 5. Percentuais médios de micronutrientes e de alumínio em relação ao conteúdo inicial do material foliar submetido a diferentes tempos de decomposição (experimento 1).

Figure 5. Mean percentages of micronutrients during decomposition process in relation to initial contents.

Fatores ambientais, da biota decompositora, da composição do próprio material e também as limitações dos métodos utilizados tornam difíceis as interpretações dos processos de decomposição em ambientes naturais. 
Considerando que a capoeira estudada situa-se em ambiente parcialmente sujeito a algumas pequenas inundações (com duração de algumas horas), podem ocorrer interferências nos processos de decomposição e liberação de elementos, embora não tenha sido registrado nenhum evento durante os experimentos. Sousa (2003), em estudo em Floresta Ombrófila Mista Aluvial, relatou que houve uma inundação no início da primavera, contaminando a serapilheira com sedimentos minerais, o que pode ter causado um aumento no teor de nutrientes detectado nos 30 primeiros dias do experimento. No entanto, o autor afirmou que o evento teve pouca influência no comportamento padrão de decomposição.

\section{CONCLUSÕES}

Para o tipo de ambiente estudado, os dados coletados indicaram um tempo médio de um ano para que a metade do material foliar caído no solo da capoeira fosse decomposto. Como em outros estudos, a perda de material foi acentuada nos primeiros meses. Essa estimativa pode ser considerada mediana entre os valores encontrados para a floresta atlântica e baixa quando comparado aos de outras florestas tropicais. Entre os macronutrientes, houve uma tendência de aumento nos teores de $\mathrm{N}, \mathrm{P}, \mathrm{Ca}$ e $\mathrm{Mg}$, e de diminuição para o K. A massa seca, o C (carbono) e o $\mathrm{Mg}$ apresentaram um comportamento similar de liberação durante o processo de decomposição. Portanto, entre os macronutrientes, o $\mathrm{K}$ e o $\mathrm{Mg}$ das folhas depositadas pela vegetação apresentam uma taxa mais rápida de ciclagem no ecossistema, sendo uma grande proporção liberada nos primeiros meses de decomposição. Já os outros macroelementos parecem ter uma interação maior no próprio local de deposição. Houve uma tendência de aumento nos teores para todos os outros elementos analisados (Fe, $\mathrm{Mn}, \mathrm{Cu}, \mathrm{Zn}$ e $\mathrm{Al}$ ) e também de aumento na quantidade de $\mathrm{Al}$ e Fe na serapilheira durante o processo de decomposição.

\section{AGRADECIMENTOS}

Agradeço ao Programa de Pós-Graduação em Engenharia Florestal da Universidade Federal do Paraná, ao PIBIC/CNPq e à CAPES, pela concessão das bolsas de estudo, ao IAPAR, pelas informações meteorológicas cedidas, aos professores Celina Wisniewski e Renato Marques, pela orientação, e aos pesquisadores Ricardo Miranda de Britez e Maísa dos Santos Guapiassú, pelo apoio prestado. Agradeço também à equipe do "Projeto Sucessão", do qual este trabalho faz parte, em especial a Gustavo Gatti, Alan Mocochinski, André Cavassani, Francisco Putini e Alexandre Lorenzetto, que também participaram diretamente da execução desta pesquisa. Este trabalho foi financiado pela Fundação O Boticário de Proteção à Natureza e apoiado pela Sociedade Fritz Müller de Ciências Naturais.

\section{REFERÊNCIAS}

ALVAREZ-SANCHEZ, J.; ENRÍQUEZ BECERRA, R. Leaf decomposition in a Mexican tropical rain forest. Biotropica, Lawrence, v. 28, n. 4b, p. 657-667, 1996.

ARUNACHALAM, A; MAITHAMI, K.; PANDEY, H. N.; TRIPATHI, R. S. Leaf litter decomposition and nutrient mineralization patterns in regrowing stands of a humid subtropical forest after tree cutting. Forest Ecology and Management, Amsterdan, v. 109, n. 1-3, p. 151-161, 1998.

BOCOCK, K. L.; GILBERT, O. J. W. The disappearance of litter under different woodland conditions. Plant and Soil, Dordrecht, v. 9, n. 2, p. 179-185, 1957.

DE VUONO, Y. S.; DOMINGOS, M.; LOPES, M. I. M. S. Decomposição da serapilheira e liberação de nutrientes na floresta da reserva biológica de Paranapiacaba, sujeita aos poluentes atmosféricos de Cubatão, São Paulo, Brasil. Hoehnea, São Paulo, v. 16, p. 179-193, 1989.

EMBRAPA. Centro Nacional de Pesquisa de solos. Sistema brasileiro de classificação de solos. Brasília, DF: EMBRAPA-SPI; Rio de Janeiro: EMBRAPA-CNPS, 1999. 412 p.

EWEL, J. J. Litter fall and leaf decomposition in a tropical forest sucession in eastern Guatemala. Journal of Ecology, Oxford, v. 64, p. 293-308, 1976. 
GAMA, D. M.; VILLLELA, D, M. Influência da fragmentação florestal na decomposição foliar de uma mata atlântica na Rebio União, RJ. In: CONGRESSO DE ECOLOGIA DO BRASIL, 6., 2003, Fortaleza, CE. Anais... Fortaleza: Editora da Universidade Federal do Ceará, 2003. p. 214-216.

GATTI, G. A. Composição florística, fenologia e estrutura da vegetação de uma área em restauração ambiental, Guaraqueçaba, PR. 114 f. Dissertação (Mestrado em Botânica) - Setor de Ciências Biológicas, Universidade Federal do Paraná, Curitiba, 2000.

GRUBB, P. J. Mineral nutrition and soil fertility in tropical rain forests. In: LUGO, A. E.; LOWE, C. (Eds.). Tropical Forests: management and ecology. New York: Springer, 1995, p. 308-330. (Ecological Studies, v. 112).

HILDEBRAND, C. Manual de análises químicas de solo e plantas. Curitiba: Editora UFPR, 1976. Mimeografado.

IAPAR - Instituto Agronômico do Paraná. Cartas climáticas de estado do Paraná - classificação segundo W. Köppen. $\quad$ Disponível em: $<$ http://www.iapar.br/Sma/Cartas_Climaticas/Classificacao_Climatica.htm>. Acesso em: 15/07/2005.

IAPAR - Instituto Agronômico do Paraná. Sistema de monitoramento agroclimático do Paraná: estação de Guaraqueçaba. $\quad$ Disponível em: $<$ http://200.201.27.14/Site/Sma/Estacoes_IAPAR/Estacoes_Parana.htm>. Acesso em: 07/03/2006.

JENNY, H.; GESSEL, S. P.; BINGHAM, T. Comparative study of decomposition rates of organic matter in temperate and tropical regions. Soil Science, Baltimore, v. 68, p. 419-432, 1949.

KLEIN, R. M. Ecologia da flora e vegetação do Vale do Itajaí (continuação). Sellowia, Itajaí, v. 32, p. 31$32,1980$.

LISANEWORK, N.; MICHELSEN, A. Litterfall and nutrient release by decomposition in three plantations compared with a natural forest in the Ethiopian lighland. Forest Ecology and Management, Amsdertam, v. 65, n.1-3, p. 149-164, 1994.

LUIZÃO, F. J. Produção e decomposição da liteira de terra firme da Amazônia Central. Aspectos químicos e biológicos da lixiviação e remoção dos nutrientes da liteira. 109 f. Dissertação (Mestrado em Ecologia) - Instituto Nacional de Pesquisas da Amazônia, Fundação Universidade do Amazonas, Manaus, 1982.

LUIZÃO, F. J.; SCHUBART, H. O. R. Produção e decomposição de liteira em floresta de terra firme da Amazônia Central. Acta Limnologica Brasiliensia, São Carlos, SP, v. 1, p. 575-600, 1987.

MAACK, R. Geografia física do estado do Paraná. Curitiba: UFPR, 1968.

MEGURO, M.; VINUEZA, G. N.; DELITTI, W. B. C. Ciclagem de nutrientes minerais na mata mesófila secundária - São Paulo. I - Produção e conteúdo de nutrientes minerais no folhedo. Boletim de Botânica, São Paulo, v. 7, p. 11-31, 1979.

MEGURO, M.; VINUEZA, G. N.; DELITTI, W. B. C. Ciclagem de nutrientes minerais na mata mesófila secundária - São Paulo. III - Decomposição do material foliar e liberação dos nutrientes minerais. Boletim de Botânica, São Paulo, v. 8, p. 7-20, 1980.

MINEROPAR MINERAIS DO PARANÁ SA. Atlasgeo.pdf: atlas geológico do estado do Paraná. Curitiba, 2001.

MOCOCHINSKI, A, Y.; WISNIEWSKI, C.; SCHEER, M. B.; PUTINI, F. A.; GATTI, G. A.; LORENZETTO, A.; CAVASSANI, A. T. Solos e vegetação em uma área em processo de regeneração ambiental em Guaraqueçaba, PR. In: CONGRESSO BRASILEIRO DE CIÊNCIA DO SOLO, 29., 2003, Ribeirão Preto, SP. Relação de trabalhos. Ribeirão Preto: UNESP: Sociedade Brasileira de Ciência Do Solo, 2003. 1 CD ROM. 
OLIVEIRA, R. R. Produção e decomposição de serrapilheira no Parque Nacional da Tijuca - Rio de Janeiro. 107 f. Dissertação (Mestrado em Geografia) - Universidade Federal do Rio de Janeiro, Rio de Janeiro, 1987.

OLSON, J. S. Energy storage and the balance of producers and decomposers in ecological systems. Ecology, Durham, v. 44, n. 2, 1963.

PALM, C. A.; SANCHEZ, P. A. Decomposition and nutrient release patterns of the leaves of three tropical legumes. Biotropica, Washington, D., v. 22, n. 4, p. 330-338, 1990.

PARANÁ. Secretaria Estadual do Meio Ambiente e Recursos Hídricos (SEMA/PR). Mapeamento da Floresta Atlântica do estado do Paraná. Curitiba, 2002. 1 CD-ROM.

PERKIN-ELMER CORPORATION. Analytical methods for atomic absorption spectrophotometry. Norwalk, 1973.

PORTES, M. C. G. de O. Deposição de serapilheira e decomposição foliar em Floresta Ombrófila Densa Altomontana, Morro Anhangava, Serra da Baitaca, Quatro Barras, PR. 90 f. Dissertação (Mestrado em Ciências Florestais) - Setor de Ciências Agrárias, Universidade Federal do Paraná, Curitiba, 2001.

PRESCOTT, C. Do rates of litter decomposition tell us anything we really need to know? Forest Ecology and Management, Amsterdam, v. 220, n. 1-3, p. 66-74, 2005.

ROCHA, A.; MARQUES, R.; CINTRA, A. P. U. Dinâmica da decomposição e liberação de nitrogênio de duas espécies arbóreas em Floresta Ombrófila Densa das terras baixas no Paraná. In: CONGRESSO DE ECOLOGIA DO BRASIL, 6., 2003, Fortaleza, CE. Anais... Fortaleza: Editora da UFC, 2003. p. 172-173.

SAMPAIO, E. V. S. B; NUNES, K. S.; LEMOS, E. Ciclagem de nutrientes na mata de Dois Irmãos (Recife - PE) através da queda de serapilheira. Pesquisa Agropecuária Brasileira., Brasília, DF, v. 23, n. 10 , p. 1055-1061, 1988.

SANTOS, V. D. Ciclagem de nutrientes minerais em mata tropical subcaducifolia nos planaltos do Paraná (Parque Estadual Vila Rica do Espírito Santo - Fênix/PR).. 385 f. Tese (Doutorado em Ecologia e Recursos Naturais) - Departamento de Ciências Biológicas, Universidade Federqal de São Carlos, São Carlos, SP, 1989.

SCHUBART, H. O. R.; FRANKEN, W.; LUIZÃO, F. J. Uma floresta sobre solos pobres. Ciência Hoje, São Paulo, v. 2, n. 10, p. 26-32, 1984.

SOUSA, S. G. A. Produção e decomposição de serapilheira de uma floresta ombrófila mista aluvial, Rio Barigui, Araucária, PR. 127 f. Tese (Doutorado em Ciências Florestais) - Setor de Ciências Agrárias, Universidade Federal do Paraná, Curitiba, 2003.

SWIFT, M. J.; ANDERSON, J. M. Decomposition. In: LIETH, H. WERGER, M. J. A. (Eds.) Tropical rain forest ecosystems: structure and function. Amsterdam: Elsevier, 1989. p. 547-569. (Biogeographical and ecological studies, n. 14 B).

TANNER, E. V. J. The decomposition of leaf litter in Jamaican montane rain forests. Journal of Ecology, Oxford, v. 69, p. 263-275.

THOMPSON, M.; VITOUSEK, P. M. Asymbiotic nitrogen fixation and litter decomposition on a long soil-age gradient in Hawaiian montane rain forest. Biotropica, Washington D.C., v. 29, n. 2, p. 134-144, 1997.

VARJABEDIAN, R.; PAGANO, S. N. Produção e decomposição de folhedo em um trecho de mata atlântica de encosta no município de Guarujá, SP. Acta Botanica Brasilica, São Paulo, v. 1, n. 2, p. 243$256,1988$. 
VELOSO, H. P.; RANGEL FILHO, A. L.; LIMA, J. C. A. Classificação da vegetação brasileira, adaptada a um sistema universal. Rio de Janeiro: IBGE, Departamento de Recursos Naturais e Estudos Ambientais, 1991.

VOGT, K. A.; GRIER, C. C.; VOGT, D. J. Production, turnover, and nutrient dynamics of above- and belowground detritus of world forests. Advances in Ecological Research, London, v. 15, p. 303-377, 1986.

WALTER, H. Vegetação e zonas climáticas. São Paulo: E. P. U., 1986.

WIEDER, R. K.; LANG, G. A critique of the analytical methods used in examining decomposition data obtained from litter bags. Ecology, Durham, v. 62, n. 6, 1982.

WISNIEWSKI, C. (Coord.) Caracterização do ecossistema e estudo das relações solo - cobertura vegetal em planície pleistocênica do litoral paranaense. Projeto Integrado - CNPq. Curitiba, UFPR, 1997. Projeto em andamento.

XULUC-TALOSA, F. J.; VESTER, H. F. M.; RAMÍREZ-MARCIAL, N.; CASTELLANOS-ALBORES, J.; LAWRENCE, D. Leaf litter decomposition pf tree species in three sucessional phases of tropical dry secondary forest in Campeche, Mexico. Forest Ecology and Management, Amsterdam, v. 174, n. 1-3, p. 401-412, 2003. 